\title{
Structure of EPCR in a non-canonical conformation
}

2

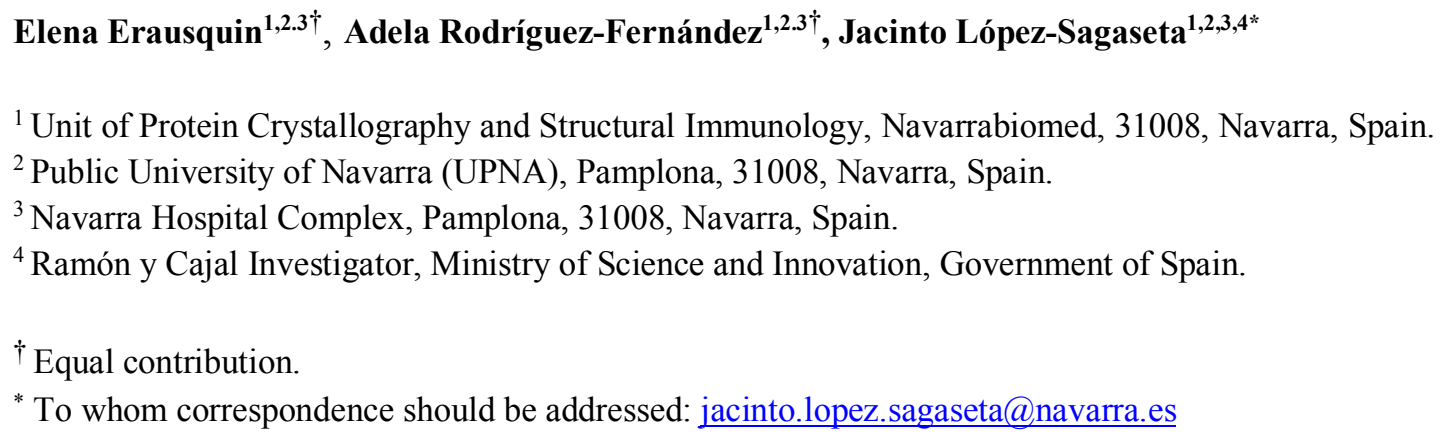

\begin{abstract}
Structural motion and conformational flexibility are often linked to biological functions of proteins. Whether the endothelial protein $\mathrm{C}$ receptor (EPCR), like other molecules, is vulnerable to folding transitions or might adopt alternative conformations remains unknown. The current understanding points to a rigid molecular structure suitable for binding of its ligands, like the anticoagulant protein $\mathrm{C}$, or the CIDR $\alpha 1$ domains of Plasmodium falciparum. In this study, we have identified a novel conformation of EPCR, captured by X-ray diffraction analyses, whereby Tyr154 shows a dramatically altered structural arrangement, likely incompatible with protein $\mathrm{C}$ binding. Biolayer interferometry analysis confirms previous results supporting a critical role for this position in protein $\mathrm{C}$ binding. Importantly, the conformational change has no apparent effect in the bound lipid. We conclude these findings reveal a site of conformational vulnerability in EPCR and inform a highly malleable region that could modulate EPCR functions.
\end{abstract}

\section{Introduction}

EPCR is an essential regulatory receptor that quickens the generation of the anticoagulant activated protein $\mathrm{C}$ (APC) on the surface of endothelial cells, which in turn prevents unhealthful levels of thrombin in blood. While EPCR mutations are linked to prothrombotic clinical outcomes ${ }^{1}$ and fetal death ${ }^{2}$ in humans, $\mathrm{EPCR}^{-/-}$mice do not survive beyond the embryonic stage due to thrombotic associated lethality ${ }^{3}$, which mirrors the critical relevance of this receptor for proper development of life in mammals. Structurally, EPCR is a non-conventional MHC class I-like protein with a well-defined molecular architecture ${ }^{4}$. Like the CD1 family of receptors, EPCR presents bound lipids in a central and hydrophobic cavity.

A high-resolution crystal structure of the protein C Gla domain bound to $\mathrm{EPCR}^{4}$, and alanine mutagenesis studies ${ }^{5}$, identified the amino acids in EPCR proximal to the Gla domain that contribute and are essential for protein C/APC binding. Among these, Tyr154 plays a critical role as it establishes a nourished network of interactions with the Gla domain that likely sustains protein C/APC binding.

Through X-ray diffraction studies, we have identified a novel, non-canonical conformational state of EPCR with a strikingly unconventional fold in the Tyr154-Thr157 
region. Particularly, a dramatic altered positioning of Tyr154 side chain is observed. This novel conformation in the $\alpha 2$ helix reveals a structurally vulnerable region in EPCR that could contribute to its varied biological functions.

\section{Results and discussion}

Structural studies performed in our laboratory enabled crystallization of EPCR in an unusual conformation (Figures $1 \mathrm{~A}-\mathrm{B}$ and $2 \mathrm{~A}$ ). Structure solution readily showed $\mathrm{F}_{\mathrm{o}}-\mathrm{F}_{\mathrm{c}}$ difference signal indicating an evident conformational change in the $\alpha 2$ helix with particular impact in the orientation of Tyr154 side chain. Previous studies by Liaw and colleagues have demonstrated that Tyr154 side chain is essential for proper binding of protein C/APC to EPCR, as Tyr154 replacement with alanine results in an EPCR form unable to bind PC/APC 5 . This non-canonical rotamer of Tyr154 is the result of an alternative structural arrangement of a short loop that switches the direction of the $\alpha_{2-1}$ helix (Figures 1A-C). The hinge-like motif can be seen as a structural piece that breaks the $\alpha 2$ helix into two independent helical rigid bodies, $\alpha_{2-1}$ and $\alpha_{2-2}$. This is a common feature also observed in MHC class I and II antigen-presenting molecules ${ }^{6}$.

Analysis of the interaction between EPCR and the protein C Gla domain shows how Tyr154 establishes numerous Van der Waals contacts with protein C Gla backbone and Asn 2 and Phe4 side chains (figure 2A). A hydrogen-bond with Gla7 further contributes to the overall network of interactions mediated by Tyr154. In this novel structure, Tyr154 shows a profound structural transition and alters the location of its side chain in a manner such that is completely away from the protein $\mathrm{C}$ binding site. The protein backbone at this region also presents a deep rearrangement, starting with a rotation of the Ala153 carbonyl by $90^{\circ}$, and followed by severe conformational shifts that affect not only Tyr 154 but Asn155, Arg156 and Thr157 peptide bonds angles and side chains (Figure 1B). Arg 156 is particularly flexible. While unliganded EPCR structures show a highly mobile Arg156 side chain, as inferred from the lack of electron density signals in previously reported structures, its position in the protein C Gla-bound structure is restricted by the interaction. This plasticity in the Arg156 side chain suggests that EPCR exists as a heterogeneous population whereby only those EPCR molecules with Arg156 in a favorable conformation enable protein C/APC binding. Or alternatively, this flexibility favors Arg 156 motion and protein $\mathrm{C}$ docking. This is consistent in our structure, where we do not observe electron density signal for Arg156 side chain. From position 156, the receptor restores its canonical conformation at Arg158.

In our crystal structure, each EPCR monomer have crystallographic symmetry mates with $\mathrm{N}$-glycosylation molecules in the vicinity of Tyr154 (Figure 2C). It could be argued that this crystal packing and crystallographic contacts might have forced this novel conformation of the $\alpha_{2-1}-\alpha_{2-2}$ loop. However, our crystal recapitulates the space group and crystal packing of the previously EPCR structure (PDB 1L8J) solved by Oganessyan et $a l^{4}$. That is, the sugar molecules near to each EPCR monomer are also present in the previously determined structure, and therefore rules out a potential crystallizationinduced conformation. Still, even if this alternative conformation was triggered by a close interacting molecule, it would reveal a site of vulnerability in a region of EPCR that is key for its anticoagulant properties. 
88

89

90

91

92

93

94

To confirm the relevance of Tyr154 in protein $\mathrm{C}$ binding, we replaced it with alanine and monitored any potential binding to EPCR. As expected, and confirming previous findings by other groups ${ }^{5,7}$, replacement of EPCR Tyr154 with alanine has a profound impact in APC binding (Figure 2B). Consequently, our results support that Tyr154 is imperative for EPCR-mediated anticoagulation.

Table 1. Diffraction data collection and refinement statistics.

$\left.\begin{array}{|c|c|}\hline \text { Resolution range } & 35.2-1.8 \\ \hline \text { Space group } & \mathrm{P} 3121 \\ \hline \text { Unit cell } & 70.4770 .4796 .649090120 \\ \hline \text { Total reflections } & 26276(2580) \\ \hline \text { Unique reflections } & 6.1(6.2) \\ \hline \text { Multiplicity } & 99.89(99.96) \\ \hline \text { Completeness (\%) } & 23.51(1.69) \\ \hline \text { Mean I/sigma(I) } & 42.16 \\ \hline \text { Wilson B-factor } & 0.0328(0.936) \\ \hline \text { R-merge } & 0.0360(1.024) \\ \hline \text { R-meas } & 0.0146(0.4107) \\ \hline \text { R-pim } & 0.999(0.665) \\ \hline \text { CC1/2 } & 0.186(0.322) \\ \hline \text { R-work } & 0.199(0.402) \\ \hline \text { R-free } & 174 \\ \hline \text { Protein residues } & 0.012 \\ \hline \text { RMS(bonds) } & 1.12 \\ \hline \text { RMS(angles) } & 96.49 \\ \hline \text { Ramachandran } \\ \text { favored (\%) }\end{array}\right)$

Values in the highest-resolution shell are in parentheses.

The lipid ligand bound in the central cavity does not show any appararent alteration. As in the previous structure with the canonical EPCR structure, the electron density maps indicate the presence of a phospholipid molecule bound in the groove in a way similar to that already found by Oganesyan et $a l^{4}$. 
101 Conformational heterogeneity and structural motion are inherent features often found in

102 proteins. For instance, G protein-coupled receptors (GPCRs) possess structural motility

103 that results essential for their biological properties ${ }^{8,9}$. Numerous crystallization studies

104 have led to a deep understanding of GPCR molecular plasticity, which results in a wide

105 spectrum of structural states. Another example of conformational plasticity is NFAT or

106 the nuclear factor of activated T cells. X-ray structural studies of this transcription factor

107 provide evidences for the coexistence of a heterogeneous population of fairly diverse

108 conformations $^{10}$.

109 In this study we have identified a novel conformation of EPCR that features a deep

110 structural motion in Tyr154 and surrounding residues. This molecular transition

111 associates with an EPCR state that lacks ability to bind protein C/APC. Our results point

112 to a multivariate folding state of EPCR in physiological conditions. The structural

113 plasticity of the hinge region represents a site of vulnerability that could be modulated by

114 alternative binders. The question remains whether this novel folding arrangement

115 represents a structural binding motif for other EPCR ligands, and which could determine

116 relevant yet unknown roles of EPCR. In this line, recent works suggest EPCR-dependent

$117 \mathrm{~T}$ cell ${ }^{11-13}$ and antibody ${ }^{14}$ recruitment, which indicates that EPCR can interact with a

118 broad variety of protein molecules.

119 In conclusion, this work reveals EPCR is not a receptor with a unique and rigid 120 conformation "ready-to-bind" its ligands. EPCR presents a structurally vulnerable region

121 in the $\alpha 2$ helix whose conformation may dictate EPCR properties in blood coagulation

122 and recognition by immune receptors.

\section{Materials and Methods}

\section{Recombinant production of EPCR}

125 The extracellular region of human EPCR was produced in sf9 insect cells. Human EPCR 126 cDNA (Genscript) was PCR amplified and cloned in frame with a GP64 signal peptide 127 in a pAcGP67A transfer vector, using BamHI and NotI restriction enzymes and 128 Optizyme $^{\text {TM }}$ T4 DNA ligase (Thermo Fisher Scientific). For crystallization purposes, the 129 EPCR construct was prepared with an N-terminal 6xHis tag followed by a 3C protease 130 cleavage site. For binding studies, we replaced this tag with a C-terminal $12 \mathrm{xHis}$ tag in a 131 new EPCR construct. The Y154A substitution was prepared using the EPCR $12 \times$ His 132 template and complementary oligos containing the desired mutation. The first PCR 133 products were used for a final overlapping PCR reaction that generated the final 134 EPCR $_{\text {Y145A }}$ construct with the C-terminal $12 x$ His motif. Sf9 insect cells $\left(\mathrm{Gibco}^{\mathrm{TM}}\right)$ were 135 transduced with the recombinant purified plasmids, BestBac $2.0 \Delta \mathrm{v}$-cath/chiA Linearized 136 Baculovirus DNA and Expres2 TR Transfection Reagent (Expression Systems) to 137 produce recombinant baculovirus. All sequences were validated by Sanger sequencing 138 (Stabvida).

\section{EPCR expression and purification}

141 Sf9 insect cells were infected with EPCR amplified baculovirus, and the culture medium 142 was collected after 72 hours of incubation at $28{ }^{\circ} \mathrm{C}$ in an orbital shaker. The culture 143 supernatant was fred of cells by centrifugation and the clarified sample was concentrated 
144 using a $10 \mathrm{KDa}$ MWCO Vivaflow (Sartorious) concentration device and dialysed 145 overnight in HBS pH 7.4 buffer before purification with a Nickel NTA Agarose 146 prepacked column (ABT). Protein was eluted with $20 \mathrm{mM}$ Hepes 7.4 supplemented with $147150 \mathrm{mM} \mathrm{NaCl}$ and $200 \mathrm{mM}$ imidazole. Purified protein was digested overnight with in148 house made $3 \mathrm{C}$ protease ${ }^{13}$ after imidazole removal. The tag-free protein was again loaded 149 into the NiNTA Agarose cartridge and the flow through recovered. All purification steps 150 were performed in an AKTA Pure 25M station (Cytiva). Protein purity was assessed by SDS-PAGE, then concentrated to $11.7 \mathrm{mg} / \mathrm{mL}$ for crystallization purposes.

\section{Crystallization and diffraction data collection}

154 EPCR $(11.7 \mathrm{mg} / \mathrm{mL})$ was first screened against different crystallization reagents (Hampton Research and Molecular Dimensions) by the sitting drop vapour diffusion method. Initial hits were optimised in 24-well plates. The largest crystals appeared in 0.1 M sodium potassium tartrate, 20\% PEG 3350 and recovered from the drop, soaked in the crystallization medium supplemented with $20 \%$ glycerol and cryo-cooled in liquid nitrogen prior to diffraction analyses.

\section{Structure determination and refinement}

162

Diffraction data was collected at the Xaloc beamline, ALBA Synchrotron (Cerdanyola del Vallès). Data was indexed and integrated with $\mathrm{XDS}^{15}$, then scaled and merged with Aimless (CCP4 crystallographic suite) ${ }^{16}$. Previously deposited coordinates of EPCR (PDB accession number 1LQV) without ligands or water molecules were used as template to solve the structure using molecular replacement with Phaser ${ }^{17}$, which was then refined with phenix.refine ${ }^{18}$. Refinement strategies included XYZ, individual B-factors and optimization of X-ray/stereochemistry weight and X-ray/ADP weight. Initial steps were performed using rigid body refinement and Translation-Libration-Screw parameters were included in the final refinement processes. Conformational changes, ligands and water molecules were added guided by $\mathrm{F}_{\mathrm{o}}-\mathrm{F}_{\mathrm{C}}$ difference maps. The final molecule was generated after several cycles of manual building in Coot and refinement.

\section{C-terminal 12xHis EPCR expression and purification}

Sf9 insect cells were infected with EPCR WT $_{\text {or EPCR }}{ }_{\text {154A }}$ baculovirus for 72 hours before culture medium collection. Culture supernatants were clarified and the proteins purified in HisGraviTrap columns (Cytiva). Proteins were eluted using $20 \mathrm{mM}$ Hepes pH 7.4, 150 $\mathrm{mM} \mathrm{NaCl}$ and $500 \mathrm{mM}$ imidazole and buffer exchanged to $20 \mathrm{mM}$ Tris $\mathrm{pH} 8.0$ on a HiPrep 26/10 Desalting column (Cytiva) before further purification on HiTrap CaptoQ ImpRes IEX column (Cytiva). Pure protein was concentrated using $10 \mathrm{KDa}$ Nanosep columns (Pall Corporation), aliquoted and frozen in liquid nitrogen for storage at $-80^{\circ} \mathrm{C}$.

\section{Biolayer interferometry}

The impact of the Y154A substitution on EPCR was assessed by biolayer interferometry using the BLItz system (Sartorius). 12xHis-tagged EPCRWT or EPCR $154 \mathrm{~A}$ was immobilized on the surface of NiNTA pre-coated biosensors (Sartorius), at a concentration of $100 \mu \mathrm{g} / \mathrm{mL}$, until stable levels were reached. The sensor was then pulsed 
with increasing concentrations of purified human APC (ThermoFisher) in $20 \mathrm{mM}$ Hepes $\mathrm{pH} 7.4,150 \mathrm{mM} \mathrm{NaCl}, 3 \mathrm{mM} \mathrm{CaCl} 2$ and $0.6 \mathrm{mM} \mathrm{MgCl}_{2}$. Interaction kinetics were calculated for each ligand and fitted to a 1:1 Langmuir binding model using the BLItz software (version 1.2.1.5).

\section{References}

1. Dennis, J. et al. The endothelial protein C receptor (PROCR) Ser219Gly variant and risk of common thrombotic disorders: a HuGE review and meta-analysis of evidence from observational studies. Blood 119, 2392-2400 (2012).

2. Franchi, F. et al. Mutations in the thrombomodulin and endothelial protein $\mathrm{C}$ receptor genes in women with late fetal loss. Br. J. Haematol. 114, 641-646 (2001).

3. Gu, J.-M. et al. Disruption of the endothelial cell protein C receptor gene in mice causes placental thrombosis and early embryonic lethality. J. Biol. Chem. 277, 43335-43343 (2002).

4. Oganesyan, V. et al. The crystal structure of the endothelial protein C receptor and a bound phospholipid. J Biol Chem 277, 24851-24854 (2002).

5. Liaw, P. C., Mather, T., Oganesyan, N., Ferrell, G. L. \& Esmon, C. T. Identification of the protein $\mathrm{C} /$ activated protein $\mathrm{C}$ binding sites on the endothelial cell protein $\mathrm{C}$ receptor. Implications for a novel mode of ligand recognition by a major histocompatibility complex class 1-type receptor. J. Biol. Chem. 276, 8364-8370 (2001).

6. Zacharias, M. \& Springer, S. Conformational flexibility of the MHC class I alpha1-alpha2 domain in peptide bound and free states: a molecular dynamics simulation study. Biophys. J. 87, 2203-2214 (2004).

7. Sampath, S. et al. Plasmodium falciparum adhesion domains linked to severe malaria differ in blockade of endothelial protein $\mathrm{C}$ receptor. Cell. Microbiol. 17, 1868-1882 (2015).

8. Mary, S. et al. Ligands and signaling proteins govern the conformational landscape explored by a G protein-coupled receptor. Proc. Natl. Acad. Sci. U. S. A. 109, 8304-8309 (2012).

9. Luttrell, L. M. \& Kenakin, T. P. Refining efficacy: allosterism and bias in G protein-coupled receptor signaling. Methods Mol. Biol. 756, 3-35 (2011).

10. Stroud, J. C. \& Chen, L. Structure of NFAT bound to DNA as a monomer. $J$. Mol. Biol. 334, 1009-1022 (2003).

11. Willcox, C. R. et al. Cytomegalovirus and tumor stress surveillance by binding of a human gammadelta $\mathrm{T}$ cell antigen receptor to endothelial protein $\mathrm{C}$ receptor. Nat. Immunol. 13, 872-879 (2012).

12. Mantri, C. K. \& St John, A. L. Immune synapses between mast cells and 
gammadelta T cells limit viral infection. J. Clin. Invest. (2018). doi:10.1172/JCI122530

13. Erausquin, E. et al. A novel $\alpha / \beta$ T-cell subpopulation defined by recognition of

14. Müller-Calleja, N. et al. Lipid presentation by the protein $\mathrm{C}$ receptor links coagulation with autoimmunity. Science 371, (2021).

15. Kabsch, W. XDS. Acta Crystallogr D Biol Crystallogr 66, 125-132 (2010).

16. Evans, P. R. \& Murshudov, G. N. How good are my data and what is the resolution? Acta Crystallogr. D. Biol. Crystallogr. 69, 1204-1214 (2013).

17. McCoy, A. J. et al. Phaser crystallographic software. J. Appl. Crystallogr. 40, 658-674 (2007).

18. Adams, P. D. et al. PHENIX: a comprehensive Python-based system for macromolecular structure solution. Acta Crystallogr D Biol Crystallogr 66, 213221 (2010).

\section{Acknowledgements}

242 This work was supported by Ramón y Cajal (RYC-2017-21683) and Generación de

243 Conocimiento (PGC2018-094894-B-I00) grants to JLS from the Ministry of Science and

244 Innovation, Government of Spain. We thank the staff of XALOC beamline at ALBA

245 Synchrotron for their assistance with X-ray diffraction data collection. We also thank

246 Maria Gilda Dichiara Rodríguez for her technical support throughout this work.

\section{Data availability}

248 Coordinates and structure factors have been deposited in the Protein Data Bank and have

249 been assigned the accession code 7Q5D.

\section{Authorship contributions}

251 Conceptualization: JLS; Experimental procedures: JLS, EEA and ARF; Data analysis:

252 JLS, EEA and ARF; Manuscript writing: JLS, EEA.

253 Disclosure of Conflicts of Interest

254 The authors declare that they have no conflict of interest.

\section{Figure Legends}

256 Figure 1. The non-canonical EPCR structure. (A) The canonical (left) and non257 canonical EPCR structures are shown in grey and paleyellow colors, respectively. The 258 residues in the $\alpha 2$ helix with severe folding transitions are highlighted. 2Fo-Fc electron 259 density maps are shown as blue meshes. In order to confirm the novel conformation, maps 260 were generated using either the canonical or non-canonical coordinates of residues 
261 Tyr154 through Thr157. The bound phospholipids are shown in the central cavities in

262 stick format (B) Superposition of the canonical and non-canonical structures. Tyr154,

263 Asn155 and Thr157 are highlighted for comparison purposes. (C) Directional switch in

264 the $\alpha 2$ helix of EPCR.

265 Figure 2. Impact of the non-canonical folding mode in protein C/APC binding. (A)

266 Structure of the protein C Gla domain in complex with the canonical conformation of

267 EPCR (PDB 1LQV). The contacts with the Gla domain established by EPCR Tyr154 are

268 highlighted with grey dashed lines. Tyr154 residues in both the canonical and non-

269 canonical EPCR structures are highlighted in sticks for comparison purposes and to better

270 visualize the impact of the folding transition in protein $C$ binding. (B) Upper panel,

271 measurement of kinetic constant rates and affinity interaction between wild type EPCR

272 and APC (upper panel). Red color traces denote buffer signal-substracted raw binding

273 data and black traces indicate fitting to a 1:1 binding kinetic model. Lower panel,

274 comparison of binding signal of $125 \mathrm{nM}$ APC to EPCR or EPCR Y154A. $_{\text {. }}$ (C) Upper panel,

275 intermolecular contacts between the non-canonical EPCR Tyr154 and N-

276 acetylglucosamine (NAG) in a crystallographic symmetry mate EPCR molecule. Lower

277 panel, analogous view with the canonical EPCR structure (PDB 1L8J). The 1L8J

278 symmetry mate (palecyan color) is shown superposed with the symmetry mate of the non-

279 canonical EPCR structure. 
A

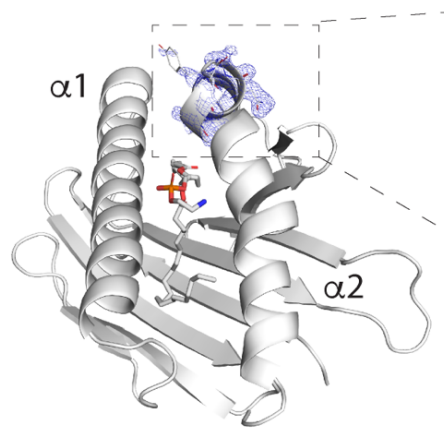

CANONICAL

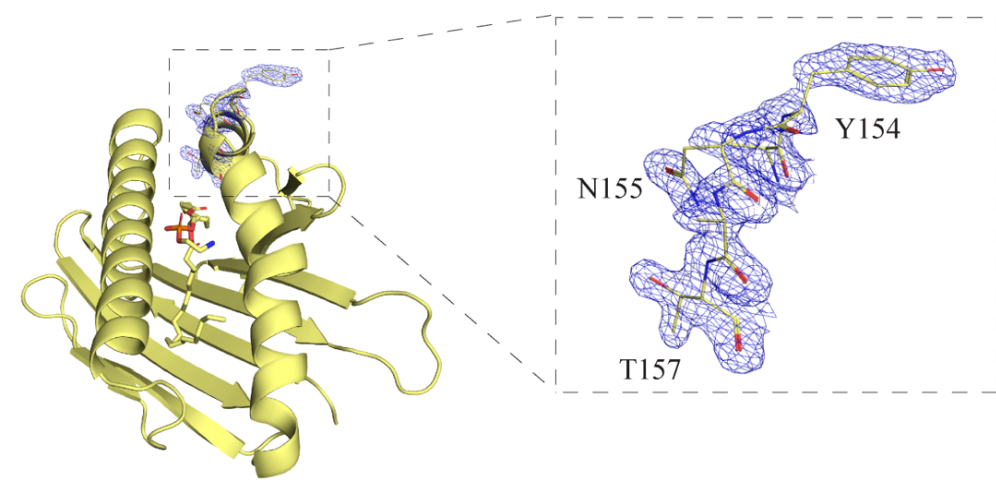

NON-CANONICAL
B

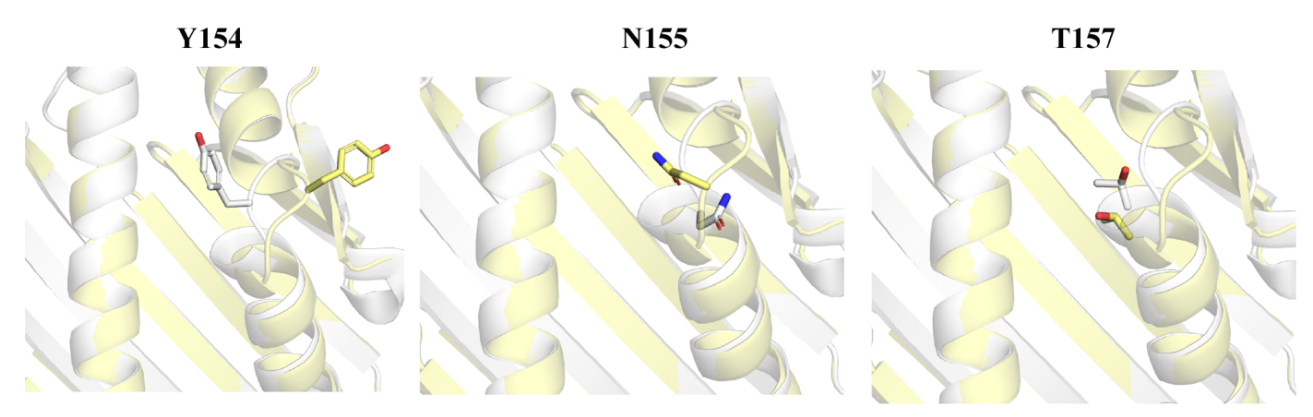

C

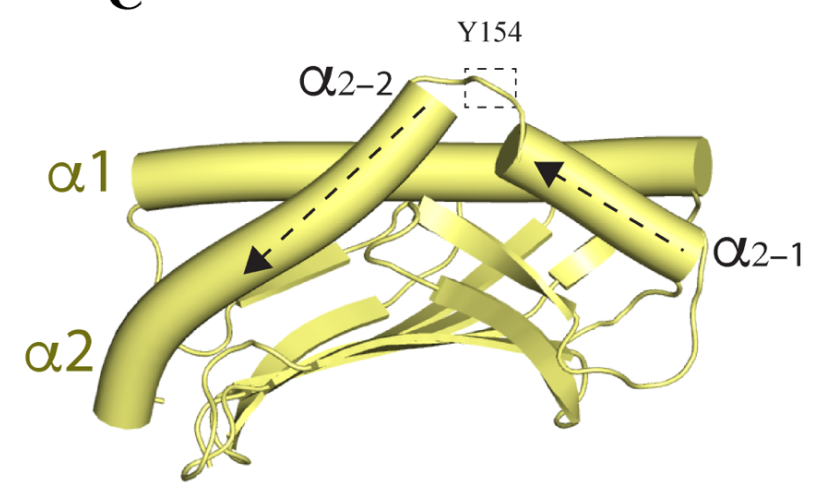


A

B

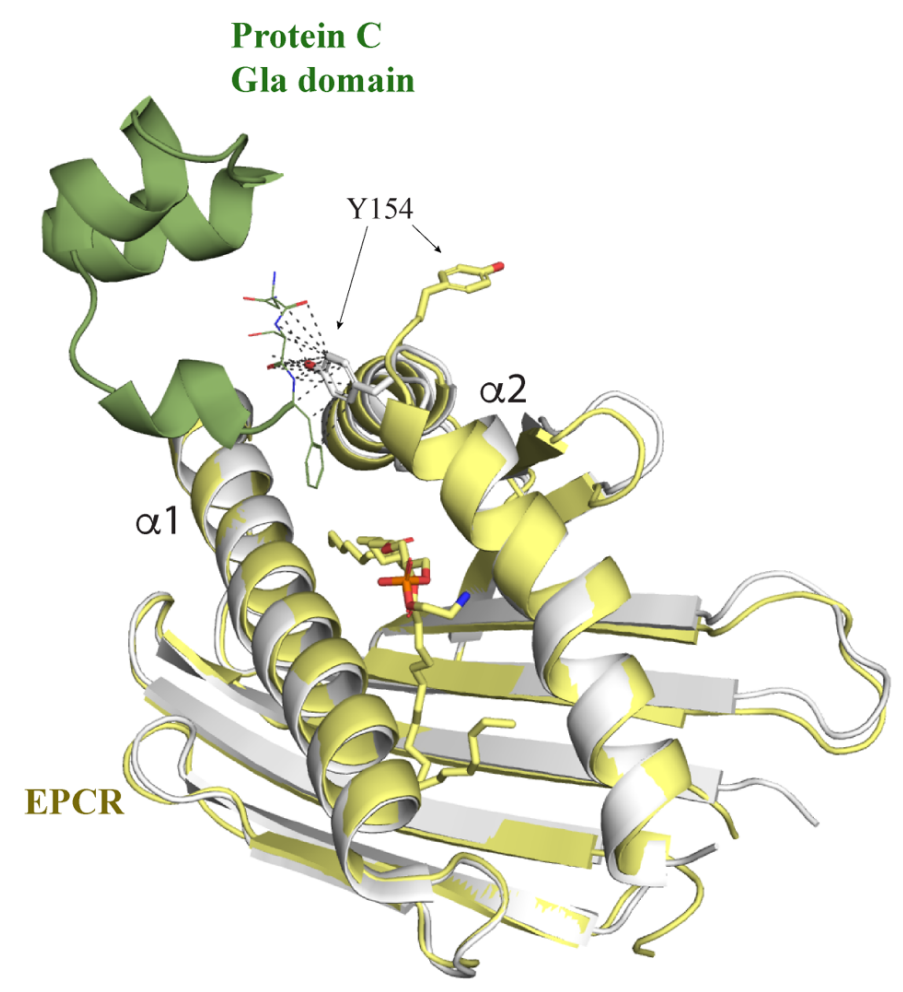

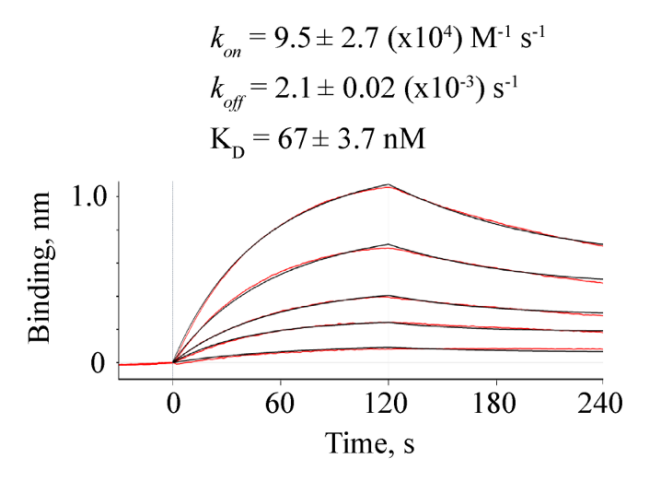

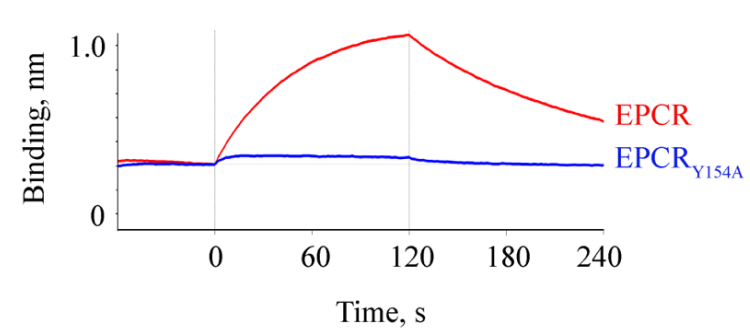

C
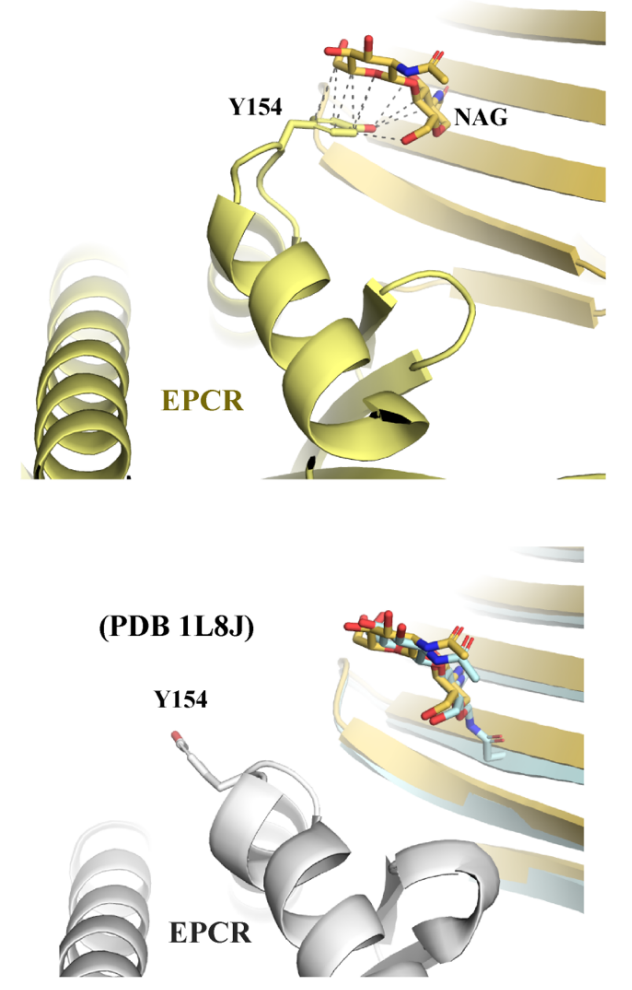